\title{
Mechanisms of Reduced Astrocyte Surface Coverage in Cortical Neuron-Glia Co-cultures on Nanoporous Gold Surfaces
}

\author{
Christopher A. R. Chapman, ${ }^{1}$ Hao Chen, ${ }^{2}$ Marianna Stamou, ${ }^{2,4}$ Pamela J. Lein, ${ }^{2}$ and Erkin Seker ${ }^{3}$ \\ ${ }^{1}$ Department of Biomedical Engineering, University of California - Davis, Davis, CA 95616, USA; ${ }^{2}$ Department of Molecular \\ Biosciences, University of California - Davis, Davis, CA 95616, USA; ${ }^{3}$ Department of Electrical and Computer Engineering, \\ University of California - Davis, 3177 Kemper Hall, Davis, CA 95616, USA; and ${ }^{4}$ Department of Health Sciences and \\ Technology, Swiss Federal Institute of Technology (ETH) Zurich, Zurich, Switzerland
}

(Received 11 February 2016; accepted 23 May 2016; published online 31 May 2016)

Associate Editor Tejal Desai oversaw the review of this article.

\begin{abstract}
Nanoporous gold (np-Au) is a promising multifunctional material for neural electrodes. We have previously shown that np-Au nanotopography reduces astrocyte surface coverage (linked to undesirable gliosis) while maintaining high neuronal coverage in a cortical primary neuron-glia coculture model as long as 2 weeks in vitro. Here, we investigate the potential influence of secreted soluble factors from cells grown on np-Au leading to the cell type-specific surface coverage on conventional tissue culture plastic. We then test
\end{abstract}

Address correspondence to Erkin Seker, Department of Electrical and Computer Engineering, University of California - Davis, 3177 Kemper Hall, Davis, CA 95616, USA. Electronic mail: eseker@ ucdavis.edu

Dr. Erkin Şeker joined the Department of Electrical and Computer Engineering at UC Davis in 2011. He received his $\mathrm{PhD}$ degree in Electrical Engineering from the University of Virginia (UVA) in 2007, where he developed techniques to control mechanical and morphological properties of nanoporous gold. During his postdoctoral appointment in the Department of Chemistry at UVA he investigated material-biomolecule interactions and developed microfluidic flow control schemes. Between 2009 and 2011, as a research associate at the Center for Engineering in Medicine at Harvard Medical School, he developed multiple electrode arrays for neural electrophysiology applications and spearheaded the development of microsystems for monitoring transcriptional and secretory dynamics at a cellular-level in the context of metabolic dysregulation. At UC Davis, he is leading the interdisciplinary Multifunctional Nanoporous Metals research group with the overarching goal of understanding and controlling nanostructured material properties and their interaction with biological systems to develop effective biomedical tools for both basic and clinical applications. He is the recipient of Fund for Medical Discovery Award from Massachusetts General Hospital and a NSF CAREER Award. He served as an Associate Scientific Advisor for Science Translational Medicine journal and was selected to participate in National Academy of Engineering's Annual Frontiers of Engineering Education Symposium in 2014.

This article is part of the 2016 Young Innovators Issue. the hypothesis that secretion of factors is responsible for inhibiting astrocyte coverage on np-Au. In order to assess whether factors secreted from cells grown on np-Au surfaces reduced surface coverage by astrocytes, we seeded fresh primary rat neuron-glia co-cultures on conventional polystyrene culture dishes, but maintained the cells in conditioned media from co-cultures grown on np-Au surfaces. After 1 week in vitro, a preferential reduction in astrocyte surface coverage was not observed, suggesting that soluble factors are not playing a role. In contrast, $4 \mathrm{~h}$ after cell seeding there were a significant number of non-adhered, yet still viable, cells for the cultures on np-Au surfaces. We hypothesize that the non-adherent cells are mainly astrocytes, because: (i) there was no difference in neuronal cell coverage between np$\mathrm{Au}$ and $\mathrm{pl}-\mathrm{Au}$ for long culture durations and (ii) neurons are post-mitotic and not expected to increase in number upon attaching to the surface. Overall, the results suggest that the $\mathrm{np}$-Au topography leads to preferential neuronal attachment shortly after cell seeding and limits astrocyte-specific np-Au surface coverage at longer culture durations.

Keywords-Nanostructure, Cell-material interaction, Nanoporous gold, Soluble factors, Cell attachment, Neuron-glia co-culture, Neuroengineering, Gliosis.

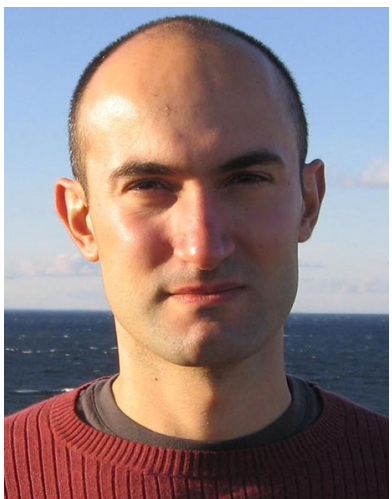




\section{INTRODUCTION}

Nanostructured materials with properties unique to the nanoscale have shown substantial promise as multifunctional coatings in a myriad of biointerfaces, ranging from orthopedic implants to neural electrodes. ${ }^{15,20,27,33}$ For example, the large effective surface area of nanostructured sensor coatings reduces impedance of neural interfaces for higher fidelity electrophysiological recordings, ${ }^{28}$ nanotubular structures allow for higher loading capacity and sustained delivery of pharmaceuticals, ${ }^{16,21}$ and nanopores act as sizeselective sieves to enhance the performance of electrochemical DNA sensors in the presence of globular biofouling proteins. ${ }^{7,25}$ In addition to these attributes, micro- and nano-topographical cues from nanostructured materials have been shown to guide cell growth, phenotype, and differentiation across many cell types, which are important factors for interfaces where specific cell coupling is beneficial. ${ }^{3,4,8,13,14}$ One emerging material that has shown promise in promoting specific cell coupling is nanoporous gold (np-Au). Np$\mathrm{Au}$ is a nanostructured material that is produced by selective dissolution of silver from a gold-silver alloy, resulting in a network of gold ligaments and pores. ${ }^{11,31}$ Specifically, np-Au has attracted attention for its applications in electrochemical sensors, ${ }^{12,22,29}$ catalytic platforms ${ }^{18}$ fundamental nanoscale materials studies, ${ }^{6,10}$ tunable drug release, ${ }^{21}$ and high fidelity recordings from organotypic brain slices. ${ }^{30}$ These properties, along with its compatibility with microfabrication techniques, suggest $\mathrm{np}-\mathrm{Au}$ as an attractive option for high sensitivity multifunctional biosensors with potential applications ranging from lab-on-a-chip based field devices to implantable drug-eluting neural electrodes. We have previously shown that np-Au nanotopography reduces the ability of astrocytes to spread across the surface, while maintaining high neuronal coverage as long as 2 weeks in vitro in a cortical primary neuron-glia co-culture model. ${ }^{5}$ Additionally, we have shown that np-Au does not alter the proliferation rate of astrocytes and therefore reduces only the hypertrophic cell surface coverage that is partially responsible for undesirable gliosis. ${ }^{26,36}$ Here, we investigate the potential influence of secreted soluble factors on the cell type-specific surface coverage as well as the initiation of this phenomenon at earlier time points after plating. Specifically, the working hypotheses are: (i) soluble factors secreted from cells on $\mathrm{np}-\mathrm{Au}$ surfaces, in response to the material nanostructure, inhibit the ability for astrocytes to spread across the np-Au surface; and (ii) reduced adhesion of astrocytes onto the np-Au surface within $4 \mathrm{~h}$ of cell plating translates into reduced surface coverage by astrocytes at longer times in culture.

\section{MATERIALS \& METHODS}

\section{Sample Fabrication and Characterization}

Samples were typically deposited in $5 \mathrm{~mm}$-diameter spots onto the middle of a piranha-cleaned $12 \mathrm{~mm}$ diameter thin $(0.15 \mathrm{~mm}$-thick) glass slide. Unstructured planar gold (pl-Au) samples were deposited by direct current sputtering (Kurt J. Lesker) of a $200 \mathrm{~nm}$-thick gold layer (at $10 \mathrm{mTorr}$ argon) on top of the substrate coated with a $160 \mathrm{~nm}$-thick chromium adhesion layer. Gold-silver alloy spots (precursor to $\mathrm{np}-\mathrm{Au}$ ) were fabricated by sputtering a $160 \mathrm{~nm}$-thick chromium adhesion layer, $80 \mathrm{~nm}$-thick gold corrosion barrier layer, and a $600 \mathrm{~nm}$-thick gold and silver alloy (64\% silver and $36 \%$ gold; at.\%). The final np-Au films were fabricated by immersing the gold-silver alloy in heated $\left(55^{\circ} \mathrm{C}\right)$ nitric acid $(70 \%)$ for $15 \mathrm{~min}$. The short dealloying times used in the present study typically result in residual silver levels in the order of $3-5$ at. $\%{ }^{5}$ The samples were then soaked in deionized (DI) water for 1 week while replacing the water every $24 \mathrm{~h}$. Caution Nitric acid is highly corrosive and reactive with organic materials and must be handled with extreme care. The morphology of the coatings was characterized by scanning electron microscopy (FEI Nova NanoSEM430), and elemental compositions before and after dealloying were assessed with energy dispersive X-ray spectroscopy (Oxford INCA, EDS).

\section{Primary Cortical Neuron-Glia Mixed Culture}

Primary rat cortical cells were obtained from the laboratory of Prof. Pamela J. Lein at the University of California, Davis. All studies were conducted according to protocols approved by the Institutional Animal Care and Use Committee of the University of California, Davis. Surface samples were incubated with $0.5 \mathrm{mg} / \mathrm{mL}$ poly-L-lysine in B Buffer (boric acid and borax, Sigma, Saint Louis, MO) at $37^{\circ} \mathrm{C}$ in $5 \% \mathrm{CO}_{2}$ for $4 \mathrm{~h}$. The samples were then washed with sterile deionized (DI) water and incubated for $12 \mathrm{~h}$ at $37^{\circ} \mathrm{C}$ and $5 \% \mathrm{CO}_{2}$ with plating media consisting of $2 \% \mathrm{~B} 27$ supplement, 1X Glutamax, 10\% heat-inactivated horse serum, and $1 \mathrm{M}$ HEPES at $\mathrm{pH} 7.5$ with Neurobasal A as the basal medium (all media components were obtained from Invitrogen, Carlsbad, CA). Dissociated cortical cells were seeded in plating medium at a density of 50,000 cells $/ \mathrm{cm}^{2}$ on each material, allowed to adhere for $4 \mathrm{~h}$, and switched to serum-free growth medium. At day in vitro (DIV) 4 and at DIV 7 cell culture medium was replenished by adding fresh growth medium. 

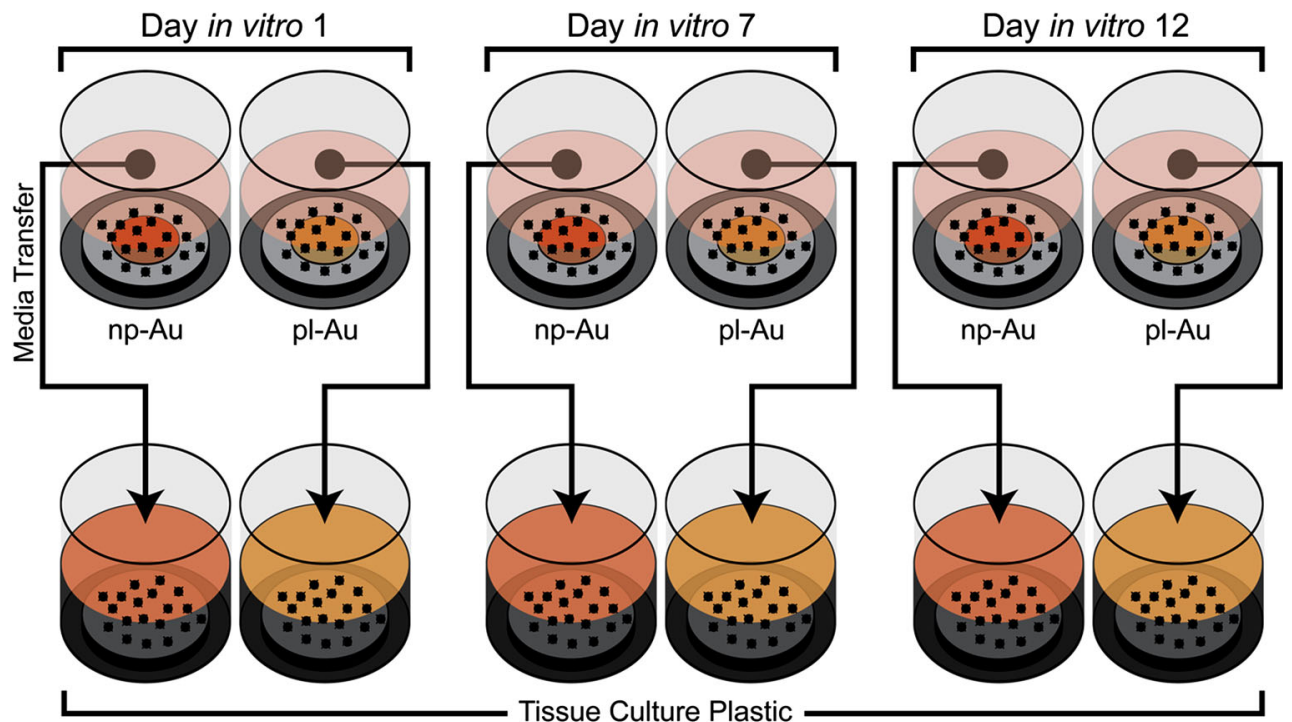

Day in vitro 7

FIGURE 1. Neuron-glia co-cultures were plated on samples of np-Au and pl-Au. These cultures were grown to day 1,7 , and 12 in vitro respectively. The conditioned media from these cultures were then used to maintain neuron-glia co-cultures on conventional polystyrene tissue culture surfaces for 7 days.

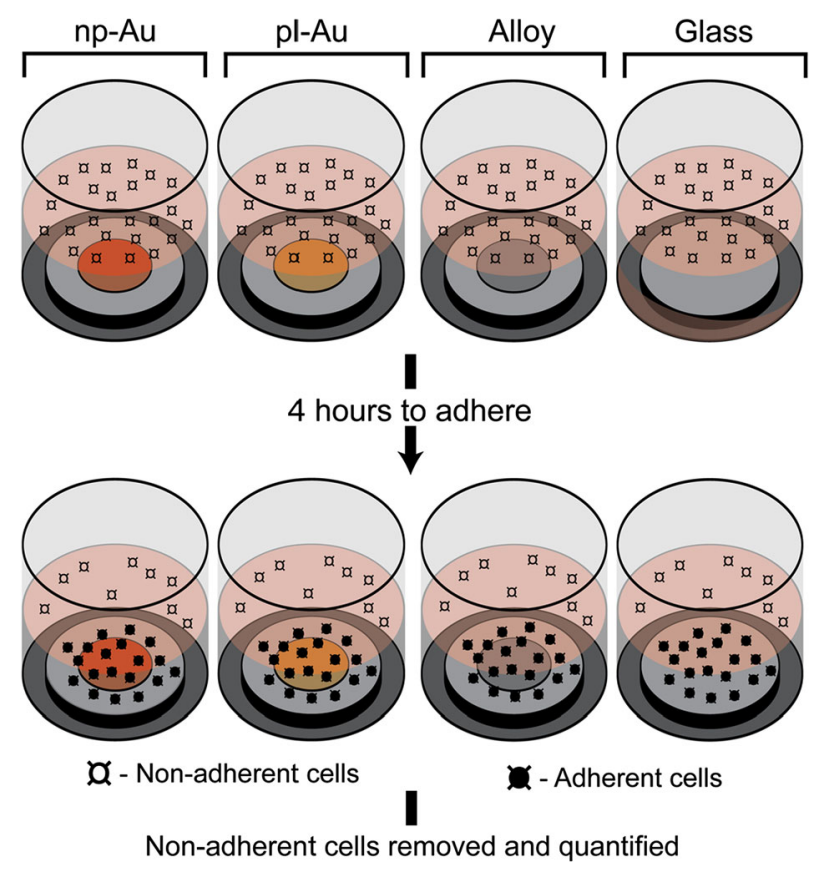

FIGURE 2. Neuron-glia co-cultures were plated on samples of $\mathrm{np}-\mathrm{Au}, \mathrm{pl}-\mathrm{Au}$, glass, and precursor Au-Ag alloy. After allowing $4 \mathrm{~h}$ for cell attachment, non-adherent cell number and viability were quantified by a trypan blue exclusion test.

\section{Primary Cortical Astrocyte Culture}

Primary rat cortical cells were obtained as previously described above. At DIV 1 the cell cultures were mechanically agitated to detach neurons from the surface of the tissue culture flask. The resulting "purified' astrocyte culture was maintained and passaged for 7 days in vitro before being plated onto substrates at a density of 50,000 cells $/ \mathrm{cm}^{2}$. Purified astrocyte cultures were maintained in a DMEM based media containing Earle's salts, $10 \%$ fetal bovine serum, and $5 \%$ penicillin/streptomycin (Invitrogen, Carlsbad, CA).

\section{Conditioned Media Experimental Protocol}

Primary rat cortical cells were seeded onto both np$\mathrm{Au}$ and pl-Au samples at a density of 50,000 cells $/ \mathrm{cm}^{2}$. Cultures were maintained on these samples for 1,7 , and 12 days respectively replenishing and changing media as per the protocol outlined above. The media from these cultures grown on $\mathrm{np}-\mathrm{Au}$ and $\mathrm{pl}-\mathrm{Au}$ was collected at the designated day (i.e., day in vitro 1, 7 , and 12) before the cultures were fixed. The collected media from each of these experiments was then used to grow fresh cultures that were plated onto conventional polystyrene tissue culture plastic at a density of 50,000 cells $/ \mathrm{cm}^{2}$. These cultures were grown to day in vitro (DIV) 7. This resulted in six different conditions being tested: conditioned media from cells cultured on np-Au surfaces to DIV 1,7 , and 12 , as well as conditioned media from cells cultured on pl-Au surfaces to DIV 1, 7, and 12 (Fig. 1). Additionally, as a control parameter non-conditioned (fresh) medium was also used to grow newly plated cultures on polystyrene surfaces to DIV 7. 
(a) np-Au (50kx)

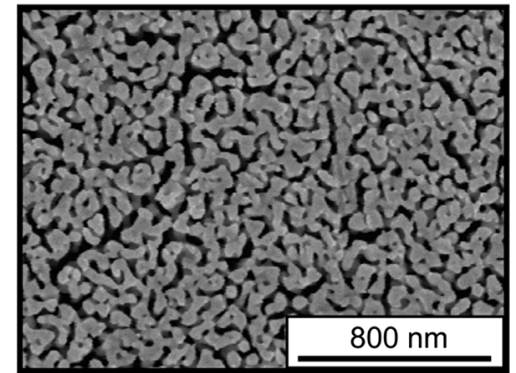

(b) np-Au (200kx)

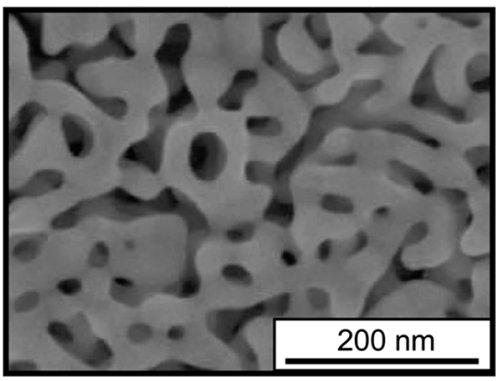

(c) pl-Au (200kx)

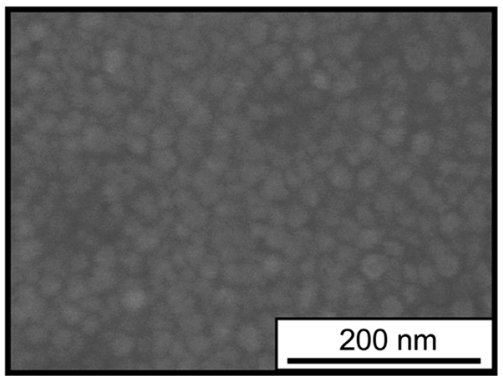

FIGURE 3. (a) A low magnification $(50,000 \times)$ scanning electron micrograph of nanoporous gold (np-Au) showing the long range uniformity of the ligament network. (b) A high magnification $(200,000 \times)$ scanning electron micrograph of $n p-$ Au showing detail of ligaments and pores. (c) A high magnification $(200,000 \times)$ scanning electron micrograph of planar gold (pl-Au) showing a clear granular surface structure.

All cultures were fixed at their designated time with $4 \%$ paraformaldehyde in phosphate buffered saline (Affymetrix), and immunostained using mouse antitubulin- $\beta$ III antibodies (Invitrogen) to identify neurons, and rabbit anti-glial fibrillary acidic protein antibodies (GFAP, Invitrogen) to identify astrocytes, followed by incubation with goat anti-mouse antibodies conjugated to Alexa Fluor 555 (Invitrogen) and goat anti-rabbit antibodies conjugated to Alexa Fluor 488 (Invitrogen) all at a concentration of 1:100. Counter-staining with DAPI was used to visualize cell nuclei. Images of immunostained samples were acquired using an inverted fluorescent microscope (Zeiss Observer D1). Sample sizes of at least three images $(10 \times$ magnification $-895 \mu \mathrm{m} \times 671 \mu \mathrm{m})$ per experimental parameter were captured and subsequently analyzed using a custom ImageJ macro to determine the total cell coverage on a given surface.

\section{Initial Cell Attachment Experimental Protocol}

Primary rat cortical cells were plated onto surfaces consisting of $\mathrm{np}-\mathrm{Au}, \mathrm{pl}-\mathrm{Au}$, precursor alloy, and glass at a density of 50,000 cells $/ \mathrm{cm}^{2}$. Cultures were given $4 \mathrm{~h}$ to adhere to each surface before the non-adherent cells were collected by extracting the medium from each sample (Fig. 2). This solution of non-adherent cells was then analyzed for cell density (number of cells) and viability through a trypan blue exclusion (ThermoFisher Scientific) test in conjunction with a hemocytometer (Brightline). This protocol was then repeated using only a purified primary astrocyte culture plated at the same density.

\section{Statistical Methods}

Each study was performed on at least three different samples per dissection, and imaged at the same five locations on each sample. Unless otherwise stated, the reported values represent averages of all samples in an experimental group and standard deviations of the measurements. A two-tailed Student's $t$ test assuming unequal variance was used to identify differences between two different sample groups and a one-way ANOVA was used when comparing more than two groups in each experiment. $p$ values less than 0.05 were deemed statistically significant.

\section{RESULTS AND DISCUSSION}

The material of focus in this study, nanoporous gold (np-Au), is obtained through a dealloying process, where silver atoms are removed from the alloy and gold atoms undergo surface diffusion creating an interconnected network of ligaments and pores. The resulting network presents a typical porosity of approximately $70 \%$ with a median ligament size of approximately $30 \mathrm{~nm}$, and non-cytotoxic levels of residual silver at $3-5$ at. $\%$ (Fig. 3). ${ }^{5}$ In a previous study we have demonstrated that the np-Au surface morphology, and not residual silver, results in reduced astrocyte (but not neuronal) surface coverage at DIV 7 and $12 .{ }^{5}$ However, the extent to which soluble factors influence cell surface coverage in the long-term as well as the short-term initiation of this phenomenon are not known. Therefore, the goal of experiments here are to determine the contribution of secreted factors to this long term reduction in astrocyte surface coverage as 
(a) Day in vitro 1 conditioned media:

Grown with media from np-Au
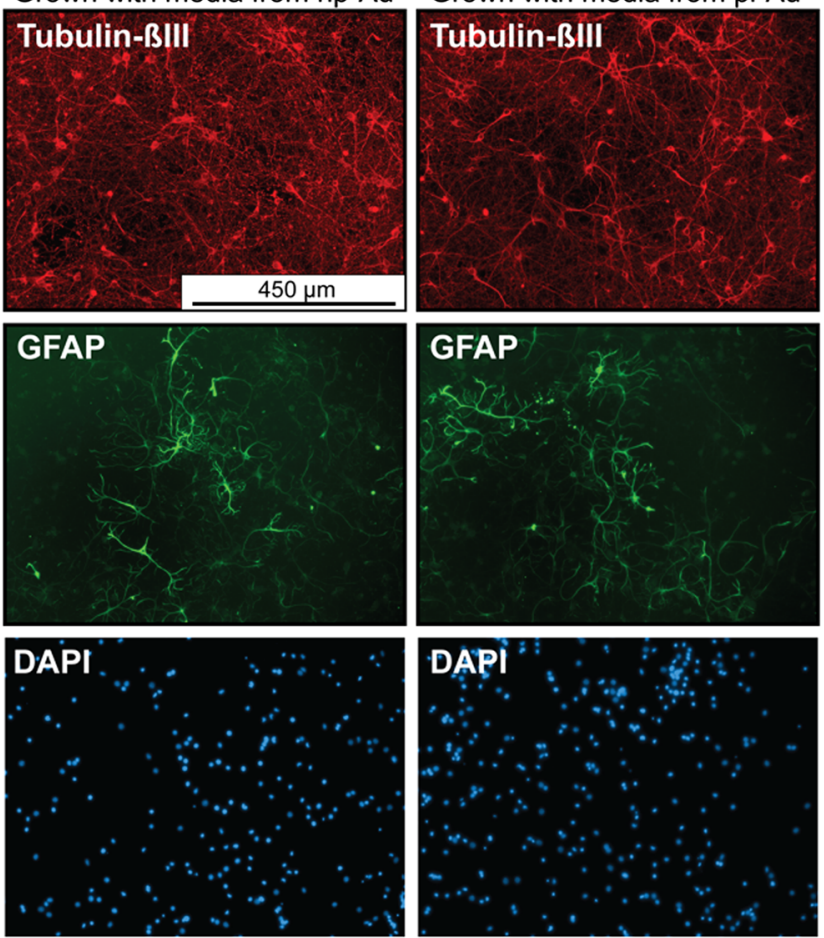

(c) Day in vitro 12 conditioned media:
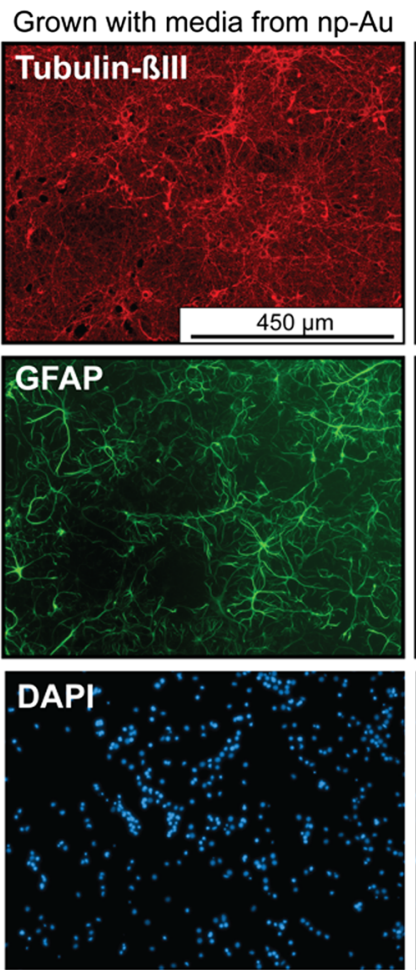
Tubulin-ßIII (b) Day in vitro 7 conditioned media:

Grown with media from np-Au
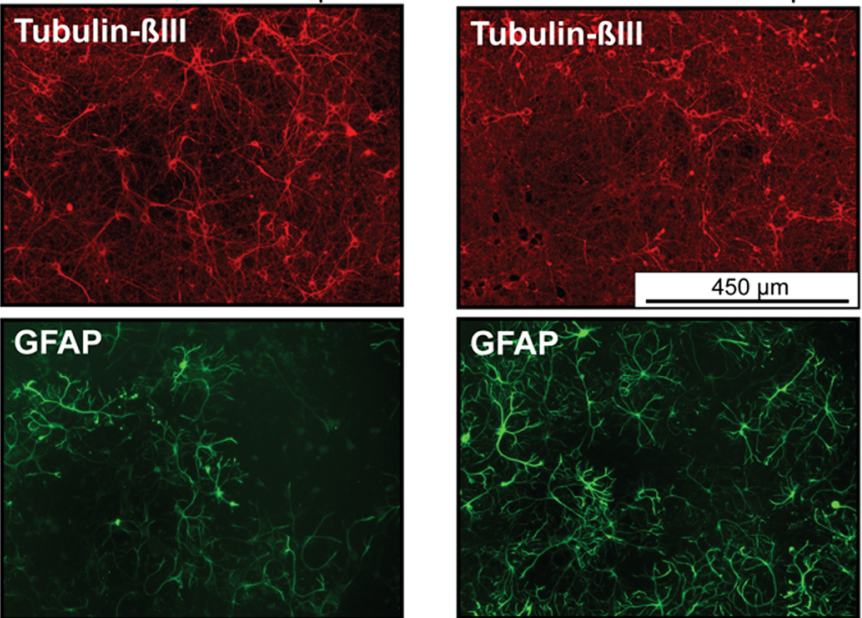

Grown with media from $\mathrm{pl-Au}$
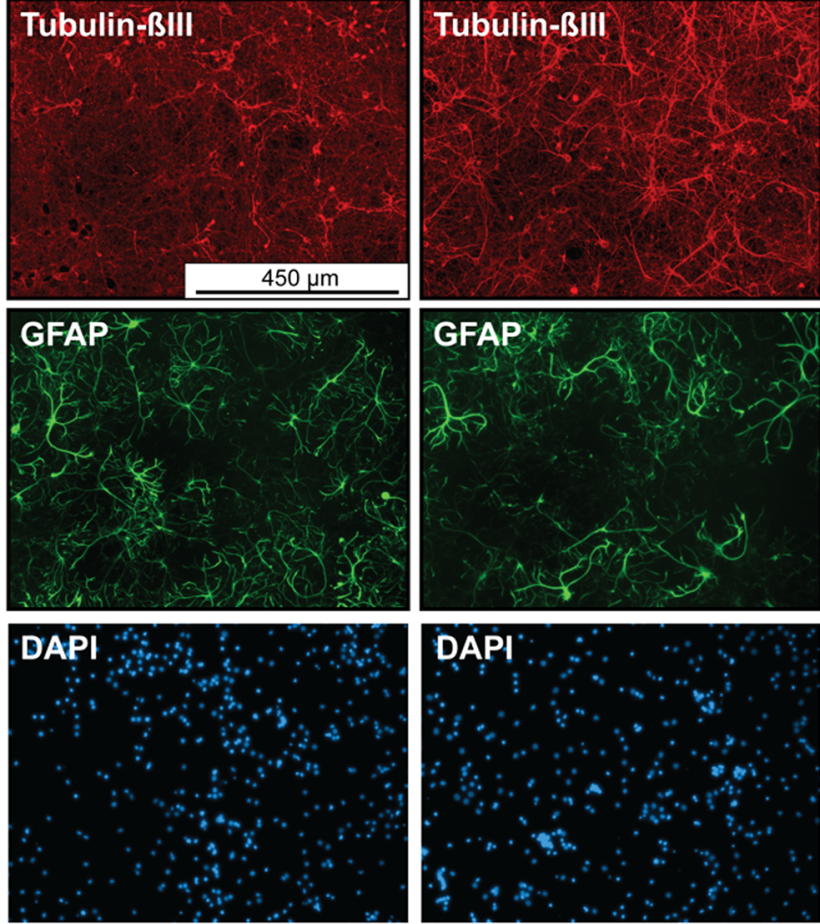

(d) Non-conditioned media (Control)

Grown with fresh media
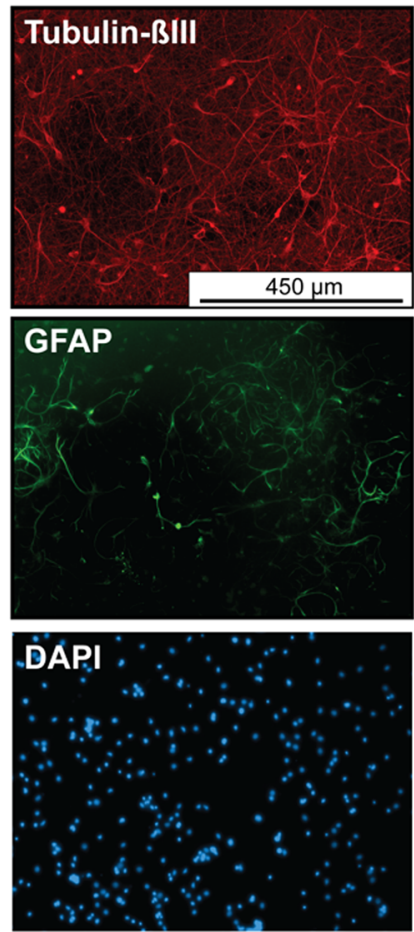

FIGURE 4. Fluorescent micrographs of cortical neurons (red-Tubulin- $\beta$ III), astrocytes (green-GFAP), and cell nuclei (blueDAPI) grown on tissue culture plastic (polystyrene) for 7 days in vitro using conditioned media from cortical cells grown on np-Au and pl-Au samples at (a) DIV 1, (b) 7, and (c) 12 as well as (d) with un-conditioned fresh media. 
(a) Neuron Surface Coverage on TCP at DIV 7

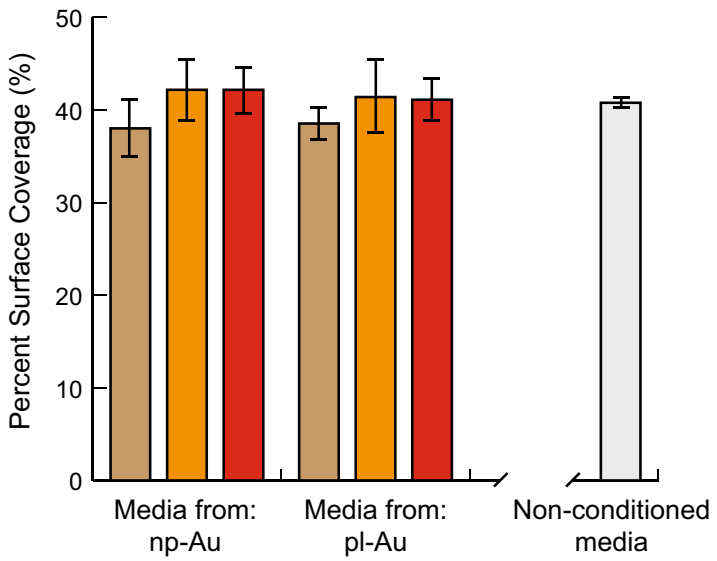

$*-p<0.05$

(c) Neuron Surface Coverage on $\mathrm{np}-\mathrm{Au}$ and pl-Au Samples

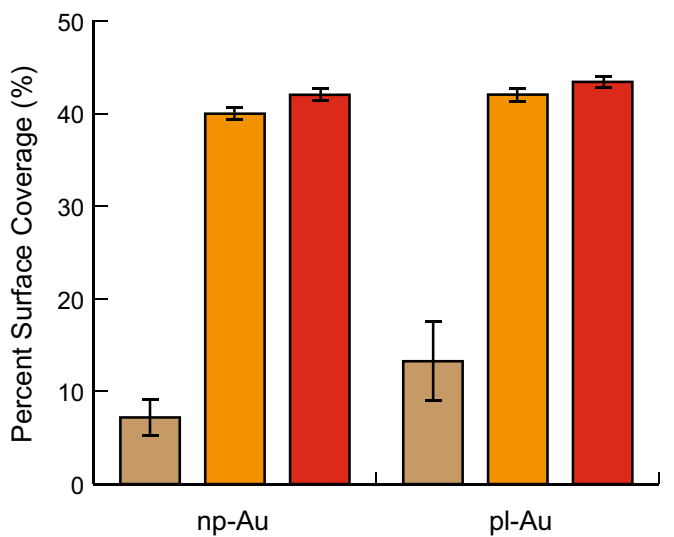

(b) Astrocyte Surface Coverage onTCP at DIV 7

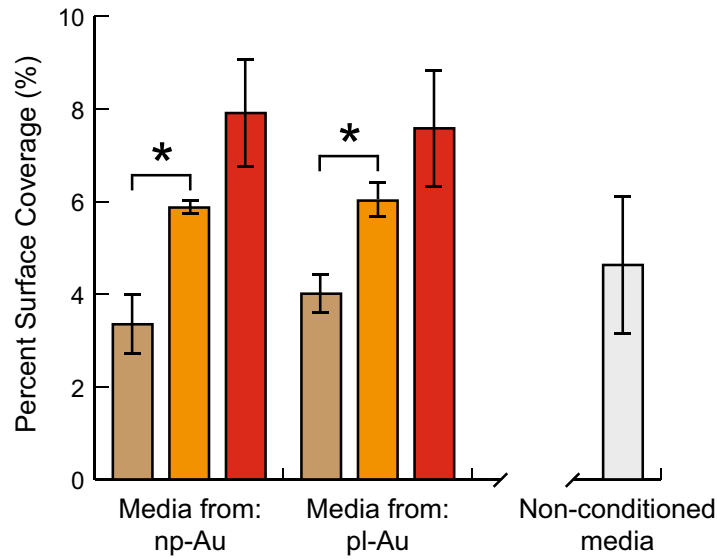

Day in vitro $12 \square$ Non-conditioned

(d) Astrocyte Surface Coverage on np-Au and pl-Au Samples

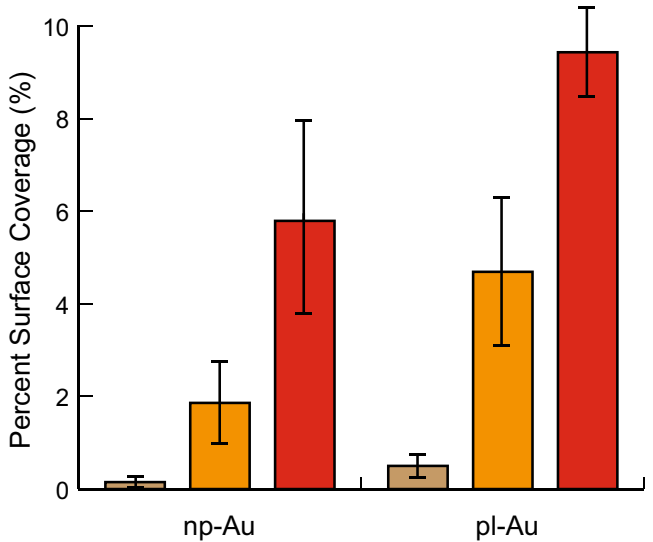

FIGURE 5. (a) Rat cortical cells were seeded on conventional culture plastic and maintained for 7 days in conditioned media from DIV 1 or DIV 7 or DIV 12 cortical cells which had been grown on np-Au or pl-Au surfaces. Plastic surface coverage by neurons on DIV 7 is not affected by the composition/age of the conditioned media $(p=0.8)$. (b) Astrocyte surface coverage at DIV 7 on polystyrene only exhibits significant increase between cultures grown with increasing age of the conditioned media from both np$\mathrm{Au}$ and $\mathrm{pl}-\mathrm{Au}(p<0.05)$, with no effect of the conditioning substrate. (c) Neuron surface coverage on np-Au and pl-Au samples at DIV 1, 7, and 12 is not different between the two sample surfaces. (d) Sustained decrease in np-Au surface coverage by astrocytes at DIV 1,7 , and 12 compared to astrocytes grown on pl-Au.

well as to determine the initial adhesive response of astrocytes to the surface of np-Au.

\section{Investigating the Role of Cell Secreted Factors on the Reduction in Astrocyte Coverage on $\mathrm{np}-\mathrm{Au}$}

Although there is strong evidence that the nanostructure of $\mathrm{np}-\mathrm{Au}$ plays a dominant effect in the reduction of astrocyte surface coverage, it is unclear whether the topographical cues directly influence spreading of astrocytes or whether factors secreted by cells cultured on $\mathrm{np}-\mathrm{Au}$ influence astrocyte spreading via an autocrine/paracrine signaling mechanism. Sol- uble cell secreted factors from neurons, astrocytes, and other glia are known to influence astrocyte surface coverage and morphology. Most notably, neurons secrete many fibroblast growth factors and glia secrete many pro-inflammatory factors such as tumor necrosis factor alpha, interleukin-6, all of which can effect astrocyte morphology and cause an increase in cell reactivity. ${ }^{1,19,32,35}$ Additionally, soluble factors from neural slice cultures have been shown to induce glioma cell proliferation. ${ }^{34}$ Given the potential influence of soluble factors in the cell type-specific reduction of astrocyte surface coverage, we test the first hypothesis concerning the role of secreted factors in reduced 
(a) np-Au - Day in vitro 12
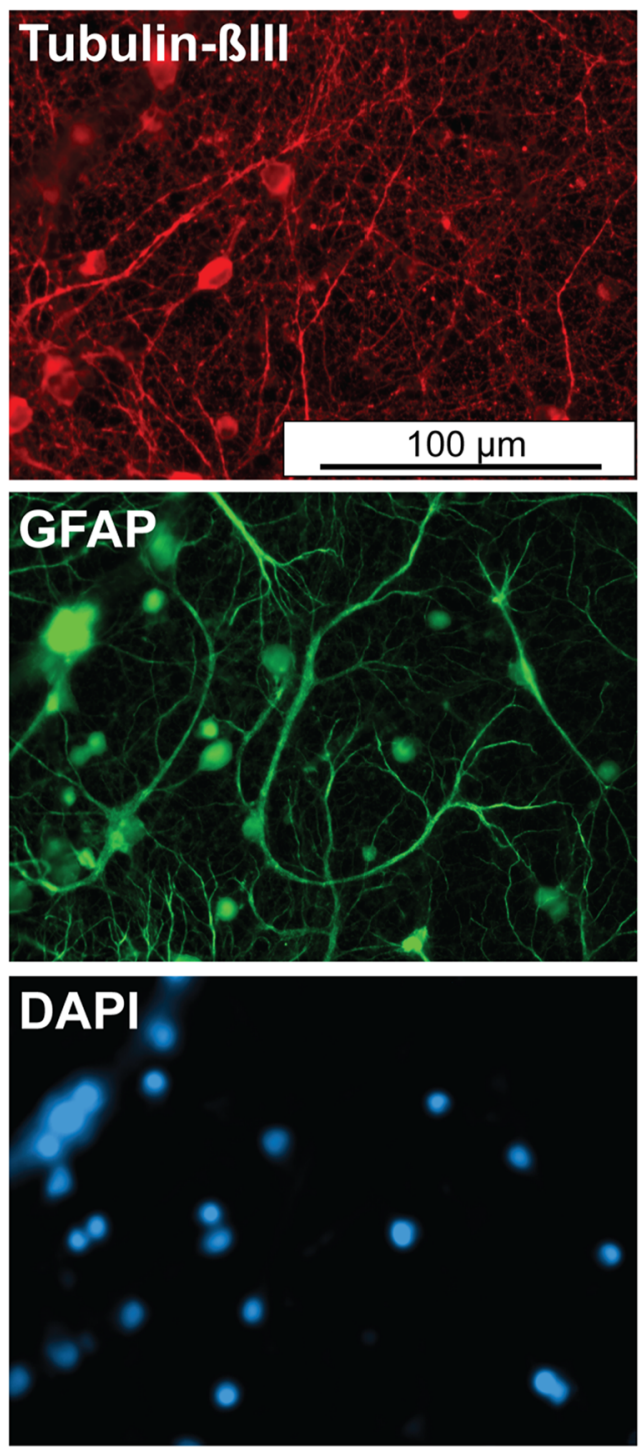

(b) pl-Au - Day in vitro 12
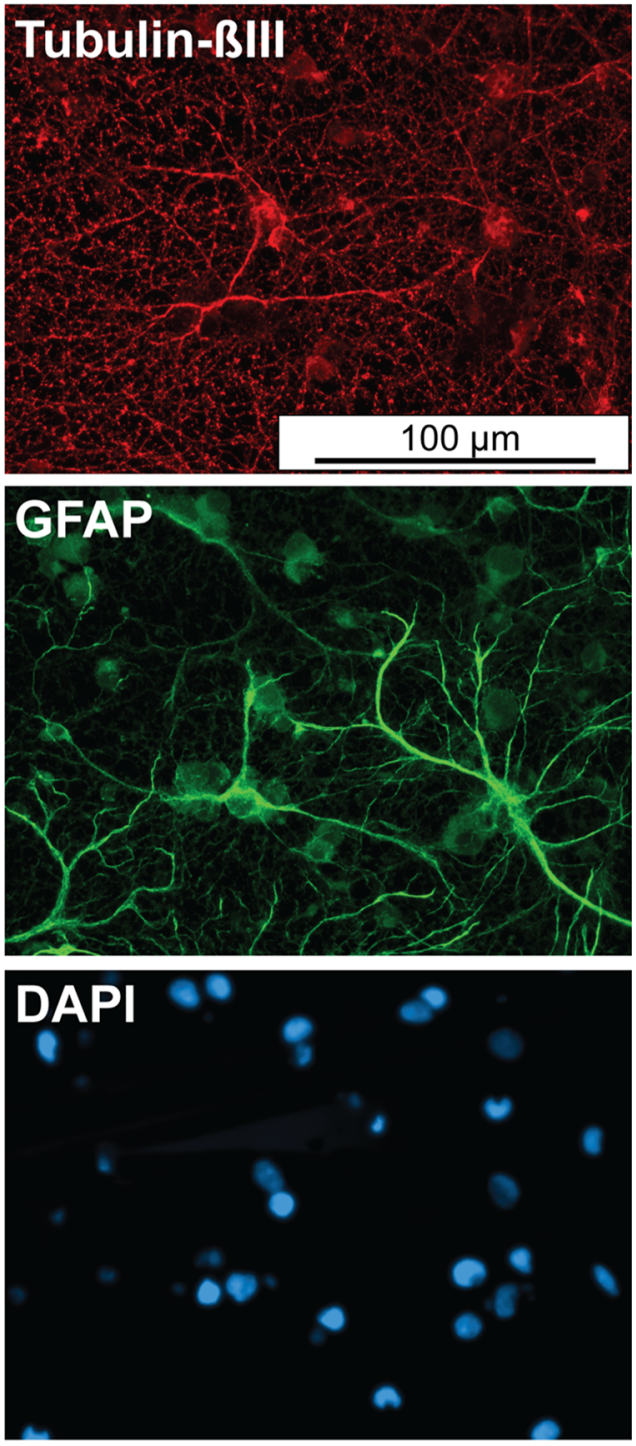

FIGURE 6. High magnification fluorescent micrographs of neurons (red-Tubulin- $\beta$ III), astrocytes (green-GFAP), and cell nuclei (blue-DAPI) grown to day in vitro 12 on (a) np-Au and (b) pl-Au.

astrocyte coverage on $\mathrm{np}-\mathrm{Au}$. In order to test this hypothesis, conditioned culture media from cortical cultures grown on np-Au and pl-Au was collected on DIV 1, DIV 7 and DIV 12. The conditioned media was then used to culture freshly harvested cortical cells on conventional polystyrene tissue culture plastic for 7 days (illustrated in Fig. 1). This experimental design ensures that the influence of soluble factors and topographical cues are decoupled.

At DIV 7, the time point at which a clear reduction in astrocyte surface coverage becomes apparent in cultures on $\mathrm{np}-\mathrm{Au},{ }^{5}$ cortical cultures grown on tissue culture plastic were immunostained with markers for neurons and astrocytes to quantify cell type-specific surface coverage in response to the different condi- tioned media (Fig. 4). The neuronal surface coverage remained similar (all samples ANOVA $p=0.3$ ) on polystyrene surfaces whether the culture was maintained in fresh (un-conditioned) medium or conditioned medium from np-Au or pl-Au cultures (Fig. 5a). In contrast, the astrocyte surface coverage monotonically increased in response to the conditioned medium from DIV 1, 7, and 12 cultures grown on $\mathrm{np}$ $\mathrm{Au}$ and pl-Au (Fig. 5b). These results suggest that the accumulation of soluble factors in conditioned media enhance proliferation and/or the spreading of astrocytes. More importantly, a pair-wise comparison between conditioned media from np-Au and $\mathrm{pl}-\mathrm{Au}$ for astrocyte surface coverage in cultures on polystyrene surfaces at DIV 7 and 12 did not reveal significant 
(a) Primary Neuron-Glia Mixed Culture

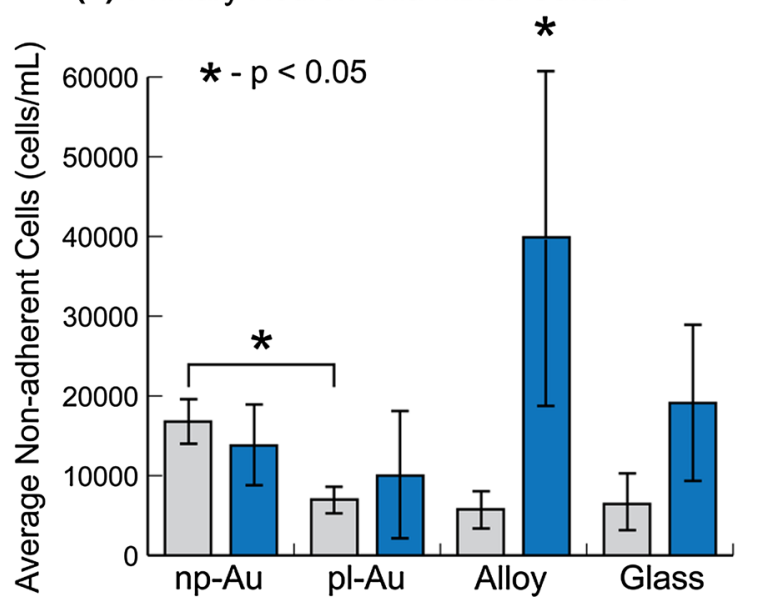

(b) Primary Purified Astrocyte Culture

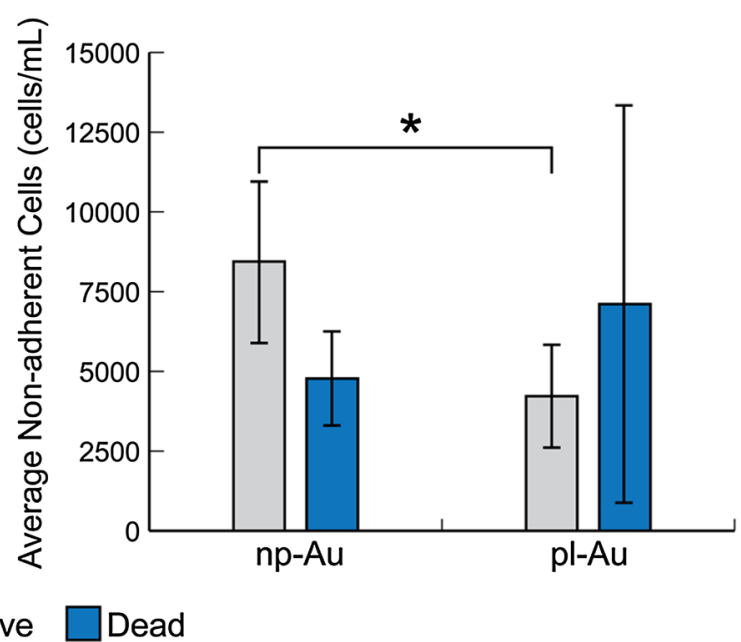

FIGURE 7. (a) A 2.4-fold increase in the number of live non-adherent cells from the np-Au surface $(p<0.005)$ in comparison to non-structured $\mathrm{pl-Au}$ and glass samples suggest that the np-Au topography reduced cell attachment via non-cytotoxic mechanisms. (b) A twofold increase in the number of live non-adherent astrocytes $(p<0.01)$ on the surface of np-Au suggests that astrocytes are the dominant cell-type in the non-adherent cortical cells.

differences. This suggests that when the topographical cues are removed (which is the case of cells grown on polystyrene with conditioned media), the astrocytespecific reduction in surface coverage is no longer present. To complement this observation, we compared the surface coverage of neurons and astrocytes on the samples (np-Au and $\mathrm{pl}-\mathrm{Au}$ ) that were used for preparing the conditioned media. A clear reduction in astrocyte surface coverage was seen on np-Au at DIV 7 and 12 (Fig. 5d), while the neuronal coverage remained unchanged (Fig. 5c). Additionally, visual comparison between high magnification images of neurons and astrocytes at DIV 12 (Fig. 6) demonstrate that clear differences in astrocyte morphology arise in response to the material surface, an effect that is not seen between conditioned media samples. Astrocyte morphologies on np-Au typically consist of fewer processes, often losing their typical 'star-shaped' cell morphology. Further studies are underway to determine whether $\mathrm{np}$-Au topography contributes to this morphological difference by affecting the local formation of focal adhesions.

\section{Effects of np-Au Surface Morphology on Initial Astrocyte Attachment}

To test the second hypothesis of whether initial cellular adhesion upon seeding dictates long-term reduction in cellular coverage, we quantified non-adherent cells and their viability in cultures grown on $\mathrm{np}-$ $\mathrm{Au}, \mathrm{pl}-\mathrm{Au}$, precursor alloy, and glass. Non-adherent cells were quantified in media collected from each sample $4 \mathrm{~h}$ after cell seeding (illustrated in Fig. 2). As shown in Fig. 7, np-Au reduces the ability of cortical cells to adhere in comparison to all surfaces. An approximately 2.4-fold increase in viable non-adherent cells $(p<0.005)$ was seen in the medium aspirated from np-Au samples in comparison to medium from pl-Au samples (Fig. 7a). Although the np-Au surface led to an increase in the number of viable non-adherent cells, there was no change in the number of dead nonadherent cells in the aspirated medium in comparison to either pl-Au or glass. On the other hand, the silverrich cytotoxic precursor alloy significantly increased the number of dead non-adherent cells $(p<0.01)$ in comparison to all other materials tested. This result, taken together with the results of the previous study that showed no change in astrocyte proliferation rates, ${ }^{5}$ suggests that $\mathrm{np}-\mathrm{Au}$ is causing an initial reduction in cortical cell adhesion via non-cytotoxic mechanisms. However, it is still unclear whether it is astrocytes or neurons that are affected by this reduction in initial cellular adhesion. To investigate the effect of surface coating on specifically the astrocytes, the cell adhesion experiments were repeated using purified astrocyte cultures from primary rat cortical cells. The number of viable non-adherent astrocytes $4 \mathrm{~h}$ after plating for the np-Au sample was twice $(p<0.01)$ that for pl-Au (Fig. 7b). Given a similar fold-increase in non-adherent cell number of cortical cells, this result suggests that the np-Au surface is non-permissive to astrocyte attachment. Surprisingly, this reduction in the initial attachment of astrocytes on np-Au does not seem to alter density or proliferation of astrocytes at later time points in culture, as previously published. ${ }^{5}$ These results suggest that the decreased cellular 
attachment to the np-Au surface is likely due to the complex nanotopography of the np-Au surface limiting adhesion sites. Additionally, astrocytes appear less likely than neurons to attach to this nanostructured surface. This can potentially be attributed to differences in focal adhesion size between astrocytes and neurons. ${ }^{2}$ It is likely that this probabilistic cell-surface interaction plays a similar role in sustained reduction in astrocyte surface coverage on $\mathrm{np}-\mathrm{Au}$ surfaces at longer time-points (past DIV 7).

\section{CONCLUSIONS}

We have decoupled putative influences of topographical and soluble cues to reveal that astrocytespecific reduction in surface coverage on $\mathrm{np}-\mathrm{Au}$ surfaces is due to both an initial inhibition of astrocyte attachment and topographical cues provided by the $\mathrm{np}-\mathrm{Au}$ nanostructure. At early time points $(4 \mathrm{~h})$ after seeding, the reduction in astrocyte adhesion on np-Au compared to $\mathrm{pl}-\mathrm{Au}$ surfaces may be attributed to kinetically-determined focal adhesion establishment during probabilistic interactions between the cellular adhesive molecules and the complex nanotopography (composed of voids and ligaments) of the np-Au surface. ${ }^{9,23}$ For later time points, when the reduced astrocyte surface coverage on $\mathrm{np}-\mathrm{Au}$ persists, the phenomenon can at least be partially attributed to the mechanical inhibition of focal adhesion complexes $^{5,14,17}$; however, it is also possible that the cell response is in part mediated via mechanotransduction by the np-Au topography through signaling pathways (such as YAP/TAZ ${ }^{24}$ ) or other integrin-mediated pathways. ${ }^{3,8}$ Further research is underway to identify the focal adhesion complex formation efficiency of astrocytes as a function of np-Au feature size. Ultimately, the results presented here demonstrate an advancement in understanding of the cellular mechanisms leading to the reduced astrocyte surface coverage on $\mathrm{np}-\mathrm{Au}$ films compared to planar materials and conventional polystyrene.

\section{ACKNOWLEDGMENTS}

We gratefully acknowledge support from UC Lab Fees Research Program Award [12-LR-237197], Research Investments in the Sciences \& Engineering (RISE) Award, and National Science Foundation Awards (CBET-1512745 and CBET\&DMR-1454426). C. Chapman was supported by a National Science Foundation Graduate Research Fellowship [DGE1148897] and a predoctoral fellowship from the Na- tional Institute of Health [T32-GM008799]. Support was also provided by the CounterACT Program, National Institutes of Health Office of the Director, and the National Institutes of Neurological Disorders and Stroke [U54-NS079202]. H. Chen was supported by a predoctoral fellowship from the National Institute of Environmental Health Sciences [T32-ES007059]. H. Chen and M. Stamou received predoctoral fellowships from the UC Davis Superfund Basic Research Program [P42-ES04699].

\section{CONFLICT OF INTEREST}

E Seker has an issued patent, titled "Nanoporous metal multiple electrode array and method of making same". C. A. R. Chapman, H. Chen, M. Stamou, and P. J. Lein declare that they have no conflicts of interest.

\section{ETHICAL STANDARDS}

All studies were conducted according to protocols approved by the Institutional Animal Care and Use Committee of the University of California, Davis. No human subjects research was performed in this study.

\section{REFERENCES}

\footnotetext{
${ }^{1}$ Agarwal, A., and D. E. Bergles. Astrocyte morphology is controlled by neuron-derived FGF. Neuron 83:255-257, 2014.

${ }^{2}$ Arregui, C., S. Carbonetto, and L. McKerracher. Characterization of neural cell adhesion sites: point contacts are the sites of interaction between integrins and the cytoskeleton in PC12 cells. J. Neurosci. 14:6967-6977, 1994.

${ }^{3}$ Biggs, M. J. P., R. G. Richards, and M. J. Dalby. Nanotopographical modification: a regulator of cellular function through focal adhesions. Nanomedicine 6:619-633, 2010 .

${ }^{4}$ Blumenthal, N. R., O. Hermanson, B. Heimrich, and V. P. Shastri. Stochastic nanoroughness modulates neuron-astrocyte interactions and function via mechanosensing cation channels. Proc. Natl. Am. Sci. 111:16124-16129, 2014. ${ }^{5}$ Chapman, C. A. R., H. Chen, M. Stamou, J. Biener, M. M. Biener, P. J. Lein, and E. Seker. Nanoporous gold as a neural interface coating: effects of topography, surface chemistry, and feature size. ACS Appl. Mater. Interfaces 7:7093-7100, 2015.

${ }^{6}$ Chapman, C. A. R., P. Daggumati, S. C. Gott, M. P. Rao, and E. Seker. Substrate toporgraphy guides pore morphology evolution in nanoporous gold thin films. Scripta Mater. 110:33-36, 2016.

${ }^{7}$ Daggumati, P., Z. Matharu, L. Wang, and E. Seker. Biofouling-resilient nanoporous gold electrodes for DNA sensing. Anal. Chem. 87:8618-8622, 2015.
} 
${ }^{8}$ Dalby, M. J., N. Gadegaard, and R. O. C. Oreffo. Harnessing nanotopography and integrin-matric interactions to influence stem cell fate. Nat. Mater. 13:558-569, 2014.

${ }^{9}$ Dembo, M., D. Torney, K. Saxman, and D. Hammer. The reaction-limited kinetics of membrane-to-surface adhesion and detachment. Proc. R. Soc. Lond. Ser. B: Biol. Sci. 234:55-83, 1988.

${ }^{10}$ Dorofeeva, T. S., and E. Seker. Electrically-tunable pore morphology in nanoporous gold thin films. Nano Res. 8(7):2188-2198, 2015.

${ }^{11}$ Erlebacher, J., M. J. Aziz, A. Karma, N. Dimitrov, and K. Sieradzki. Evolution of nanoporosity in dealloying. Nature 410:450-453, 2001

${ }^{12}$ Feng, J., W. Zhao, and J. Wu. A label-free optical sensor based on nanoporous gold arrays for the detection of oligodeoxynucleotides. Biosens. Bioelectron. 30:21-27, 2011.

${ }^{13}$ Gasiorowski, J. Z., S. J. Liliensiek, P. Russel, D. A. Stephan, P. A. Nealey, and C. J. Murphy. Alterations in gene expression of human vascular endothelial cells associated with nanotopographic cues. Biomaterials 31:8882-8888, 2010.

${ }^{14}$ Geiger, B., J. P. Spatz, and A. D. Bershadsky. Environmental sensing through focal adhesions. Nat. Rev. Mol. Cell Bio. 10:21-33, 2009.

${ }^{15}$ Goldberg, M., R. Langer, and X. Jia. Nanostructured materials for applications in drug delivery and tissue engineering. J. Biomater. Sci. Polym. Ed. 18:241-268, 2007.

${ }^{16}$ Gultepe, E., D. Nagesha, S. Sridhar, and M. Amiji. Nanoporous inorganic membranes or coatings for sustained drug delivery in implantable devices. Adv. Drug Del. Rev. 62:305-315, 2010.

${ }^{17}$ Jeon, H., S. Koo, W. M. Reese, P. Loskill, C. P. Grigoropoulos, and K. E. Healy. Directing cell migration and organization via nanocrater-patterned cell-repellent interfaces. Nat. Mater. 14:918-923, 2015.

${ }^{18} \mathrm{Jin}$, H. J., and J. Weissmuller. A material with electrically tunable strength and flow stress. Science 332:1179-1182, 2011.

${ }^{19}$ Kang, K., S. Lee, J. E. Han, J. W. Choi, and M. Song. The complex morphology of reactive astrocytes controlled by fibroblast growth factor signaling. Glia 62:1328-1344, 2014.

${ }^{20}$ Kotov, N. A., J. O. Winter, I. P. Clements, E. Jan, B. P. Timko, S. Campidelli, S. Pathak, A. Mazzatenta, C. M. Lieber, M. Prato, R. V. Bellamkonda, G. A. Silva, N. W. S. Kam, F. Patolsky, and L. Ballerini. Nanomaterials for neural interfaces. Adv. Mater. 21:3970-4004, 2009.

${ }^{21}$ Kurtulus, O., P. Daggumati, and E. Seker. Molecular release from patterned nanoporous gold thin films. Nanoscale 6:7062-7071, 2014.

${ }^{22}$ Li, K., J. Huang, G. Shi, W. Zhang, and L. Jin. A sensitive nanoporous gold-based electrochemical DNA biosensor for Escherichia coli detection. Anal. Lett. 44:2559-2570, 2011.

${ }^{23}$ Mody, N. A., and M. R. King. Influence of Brownian motion on blood platelet flow behavior and adhesive dynamics near a planar wall. Langmuir 23:6321-6328, 2007.

${ }^{24}$ Morgan, J. T., C. J. Murphy, and P. Russell. What do mechanotransduction, Hippo, Wnt, and TGFB have in common? YAP and TAZ as key orchestrating molecules in ocular health and disease. Exp. Eye Res. 115:1-12, 2013.

${ }^{25}$ Patel, J., L. Radhakrishnan, B. Zhao, B. Uppalapati, R. C. Daniels, K. R. Ward, and M. M. Collinson. Electrochemical properties of nanostructured porous gold electrodes in biofouling solutions. Anal. Chem. 85:11610-11618, 2013.

${ }^{26}$ Polikov, V. S., P. A. Tresco, and W. M. Reichert. Response of brain tissue to chronically implanted neural electrodes. $J$. Neurosci. Methods 148:1-18, 2005.

${ }^{27}$ Popat, K. C., L. Leoni, C. A. Grimes, and T. A. Desai. Influence of engineered titania nanotubular surfaces on bone cells. Biomaterials 28:3188-3197, 2007.

${ }^{28}$ Robinson, D. A. The electrical properties of metal mircoelectrodes. Proc. IEEE 56:1065-1071, 1968.

${ }^{29}$ Santos, G. M., F. Zhao, J. Zeng, and W. Shih. Characterization of nanoporous gold disks for photothermal light harvesting and light-gated molecular release. Nanoscale 6:5718-5724, 2014.

${ }^{30}$ Seker, E., Y. Berdichevsky, M. R. Begley, M. L. Reed, K. J. Staley, and M. L. Yarmush. The fabrication of low-impedance nanoporous gold multiple-electrode arrays for neural electrophysiology studies. Nanotechnology 21:1-7, 2010.

${ }^{31}$ Seker, E., M. L. Reed, and M. R. Begley. Nanoporous gold: fabrication, characterization, and applications. $M a-$ terials 2:2188-2215, 2009.

${ }^{32}$ Sofroniew, M. V. V., and H. V. Vinters. Astrocytes: biology and pathology. Acta Neuropathol. 119:7-35, 2010.

${ }^{33}$ Solanki, P. R., A. Kaushik, V. V. Agrawal, and B. D. Malhotra. Nanostructured metal oxide-based biosensors. NPG Asia Mater. 3:17-24, 2010.

${ }^{34}$ Venkatesh, H. S., T. B. Johung, V. Caretti, A. Noll, Y. Tang, S. Nagaraja, E. M. Gibson, C. W. Mount, J. Polepalli, S. S. Mitra, P. J. Woo, R. C. Malenka, H. Vogel, M. Bredel, P. Mallick, and M. Monje. Neuronal activity promotes glioma growth through neuroligin-3 secretion. Cell 161:803-816, 2015.

${ }^{35}$ Wagoner, N. J., J. Oh, P. Repovic, and E. N. Benveniste. Interleukin-6 production by astrocytes: autocrine regulation by IL-6 and the soluble IL-6 receptor. J. Neurosci. 19:5236-5244, 1999.

${ }^{36}$ Woolley, A. J., H. A. Desai, and K. J. Otto. Chronic intracortical microelectrode arrays induce non-uniform, depth-related tissue responses. J. Neural Eng. 10:1-11, 2013. 